\title{
Salvage of Free Jejunum Flap Following Late Venous Compromise
}

\section{George Virich, Riyadh Almasharqah, Allessandra Canal, Charles Malata*}

Department of Plastic and Reconstructive Surgery, Addenbrooke’s Hospital, Cambridge University Teaching Hospitals NHS Foundation Trust, Cambridge, UK.

Email: *charles.malata@addenbrookes.nhs.uk

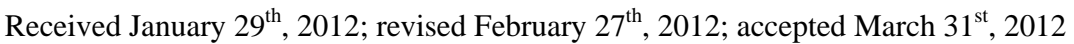

\begin{abstract}
Single stage reconstruction of circumferential pharyngo-esophageal defects using the free jejunum flap produces excellent functional results with minimal morbidity and mortality. The most serious complication with this flap is vascular compromise, usually within the first 24 - 48 hours after surgery. Compromised flaps are frequently diagnosed late often making their salvage impossible, so close postoperative monitoring of tissue perfusion is critical. Rapid identification and salvage of the failing flap increases the chance of a successful outcome.
\end{abstract}

Keywords: Flap; Free; Microvascular; Pharyngo-Oesophagectomy; Jejunum; Complications; Thrombosis; Venous; Salvage

\section{Introduction}

Single stage reconstruction of circumferential pharyngooesophageal defects using the free jejunum flap is an established technique [1]. It commonly produces excellent functional results with minimal morbidity and mortality [2-5]. The most serious complication with this flap is vascular compromise, namely inadequate venous drainage (85\%) or arterial supply (15\%) [6]. Sufficient venous drainage is the most critical factor for a successful free jejunal transfer. Between five and ten percent of patients develop vascular compromise, usually within the first 24 - 48 hours after surgery, which may culminate in partial or even complete flap loss [6]. Monitoring of jejunal flaps is difficult, and therefore compromised flaps are frequently diagnosed late often making their salvage impossible [7]. Therefore, close postoperative monitoring of tissue perfusion is critical [8,9]. Furthermore, rapid identification and salvage of the failing flap increases the chance of a successful outcome while delay causes tissue necrosis, a disaster for both the patient and the surgeon [10].

A 72 year old female treated for a post-cricoid squamous cell carcinoma by pharyngo-oesophagectomy and free jejunum flap transfer developed a late (post-operative day 5) thrombosis of the drainage vein. The flap compromise was recognized early and rescued by immediate re-exploration and re-anastomosis.

\section{Case Report}

A 72-year-old female smoker with a background history of

${ }^{*}$ Corresponding author. essential thrombocytopenia and hypertension presented with voice changes characterised by hoarseness in September 2004. By December 2004 she had developed dysphagia for both solids and liquids complicated by several episodes of choking. Barium swallow suggested a post-cricoid oesophageal web (Figure 1).

Initially, upper gastrointestinal endoscopy and dilation was unsuccessful at improving the dysphagia. A second endoscopy under screening revealed an ill-defined fleshy mass at the level of cricopharyngeus not consistent with a simple oesophageal web. Panendoscopy and biopsy of

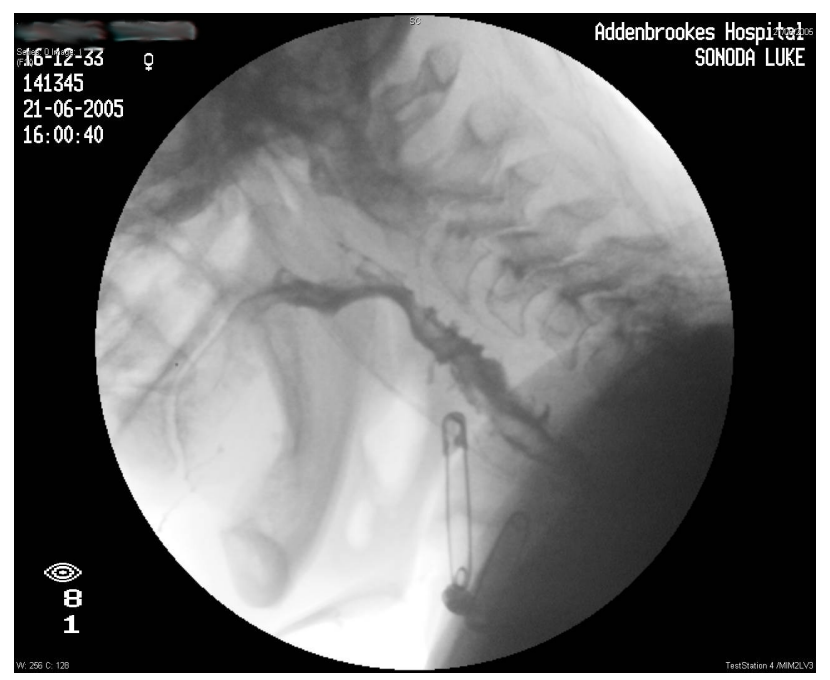

Figure 1. Barium swallow suggestive of a post-cricoid oesophageal web. 
the mass was performed. As part of the staging process she underwent a Computer Tomography (CT) scan of neck and thorax and this was suggestive of mediastinal lymphadenopathy. Histology confirmed squamous cell carcinoma of the post-cricoid region extending into the cervical oesophagus.

She underwent palliative surgery in June 2005 comprising laryngopharyngectomy, upper oesophagectomy, and reconstruction using a free jejunum flap. The flap's artery and vein were anastomosed to their recipient vessels end to end and end to side respectively.

Postoperatively the flap was monitored through a window using a combination of clinical and non-invasive instrumental methods. Flap's colour, capillary refill, peristalsis, bleeding after a needle prick, and temperature (using a temperature probe) were monitored hourly. The flap was observed to be pink, moist, with normal peristalsis during the first three days. On day four no peristalsis was observed, but the flap remained pink and moist. On day five the patient developed haematemesis producing approximately one litre of fresh blood from the back of the throat during the afternoon. A clinical decision was made to return the patient to theatre urgently, perform flexible oesophagogastroduodenoscopy (OGD), and re-explore the flap.

OGD demonstrated a congested ischaemic flap mucosa. No specific bleeding points were observed, and only minimal oozing from one end of the flap was observed. Re-exploration of the right side of the neck revealed a grossly congested flap mesentry resembling a large haematoma. There was still a good arterial inflow but a thrombosed vein that was collapsed distal to the anastomosis. Revision of the vein through end-to-side anastomosis to internal jugular vein produced good venous outflow. Flap congestion improved dramatically and peristalsis of the jejunum returned to normal. Postoperatively the patient had an uneventful recovery and was discharged home after three weeks with an intact flap with normal function.

A few months later she received a six weeks course of radiotherapy. At insertion of percutaneous endoscopic gastrostomy (PEG) tube to facilitate feeding during radiotherapy the gastroscopy revealed a healthy jejunum interposition flap and a barium swallow demonstrated no leak of contrast (Figure 2). Similarly, barium swallow performed ten months after the original surgery demonstrated no leak and good flow of contrast medium into the stomach (Figures 3 and $\mathbf{4}$ ).

\section{Discussion}

Venous occlusion secondary to thrombosis in jejunal free flap is an uncommon but critical complication. To reduce the reliance on the main vein and the chance of venous

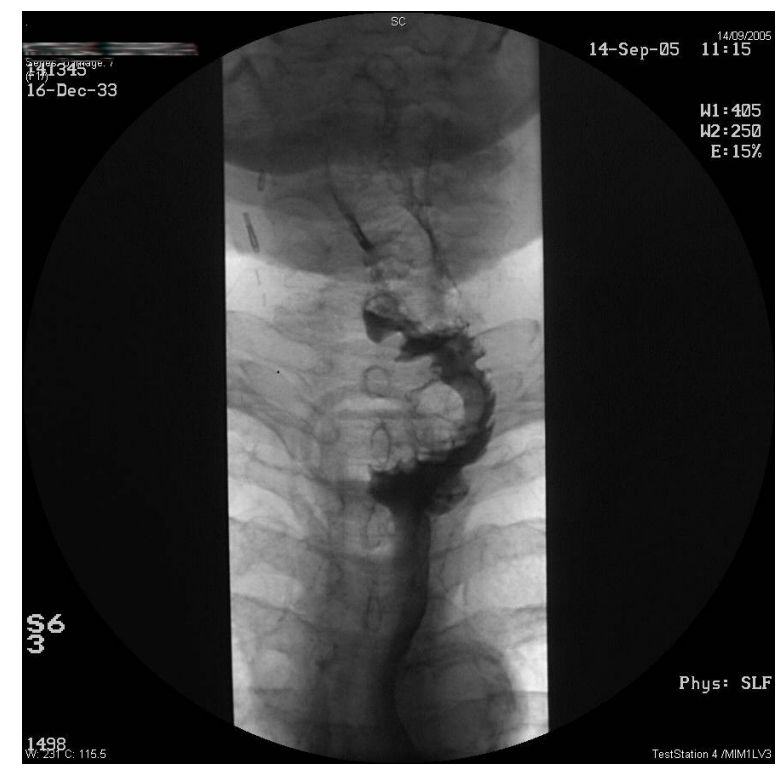

Figure 2. Barium swallow three months postoperatively demonstrating no leak of contrast.

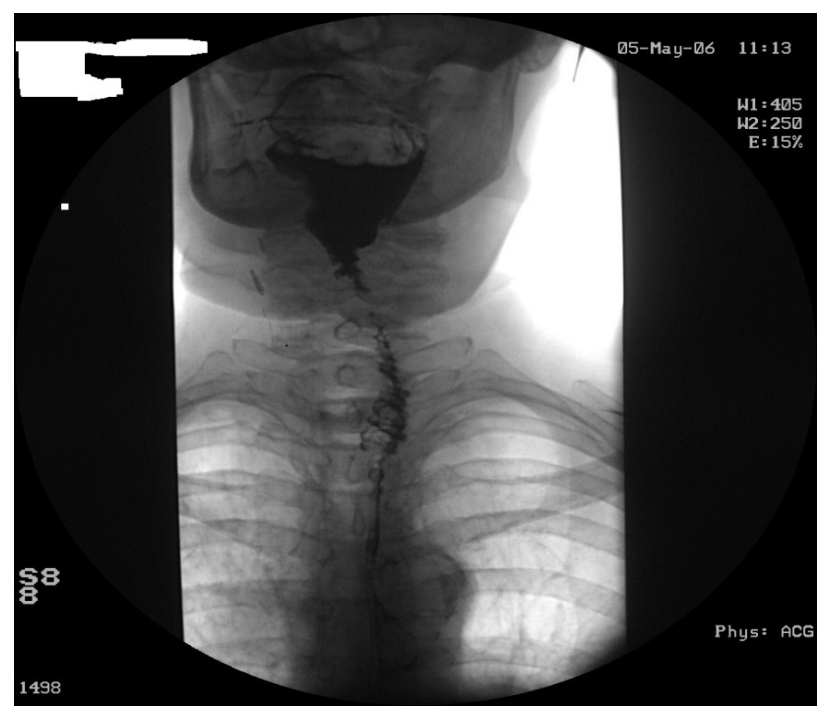

Figure 3. Barium swallow (anterior-posterior view) ten months post op demonstrating no leak and good flow of contrast medium into the stomach.

outflow compromise, "supercharging" of the flap has been used [11]. Although this can help a struggling flap, most of the drainage remains via the pedicle vein. Therefore close monitoring of flap's vascular status is essential since flap repair after segmental necrosis has poorer functional outcome than the uncomplicated reconstruction.

Several important points emerge from this case. First, vascular insufficiency can occur late, i.e. after the "high risk” 24 - 48 hours postoperative period. During the first seven days re-establishment of axial blood supply is essential for flap survival, whereas after seventeen days, 


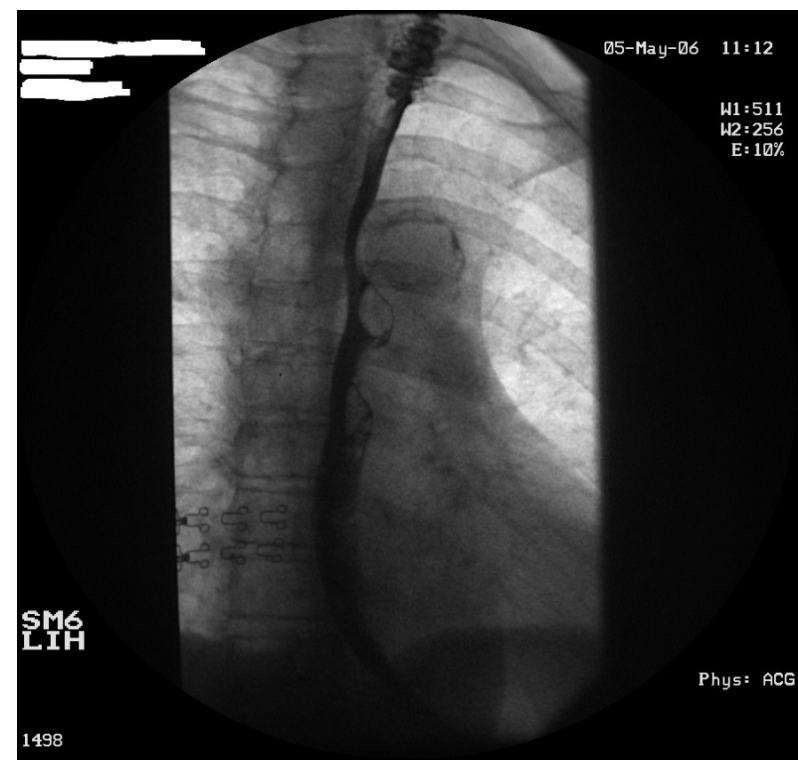

Figure 4. Barium swallow (lateral view) ten months post op demonstrating no leak and good flow of contrast medium into the stomach.

vascularisation from the recipient bed is said to be adequate to maintain viability [12]. Based on these observations early re-exploration of the pedicle approach to flap salvage is recommended, and in our case the pedicle was successfully re-explored on postoperative day five. Second, endoscopic evaluation can aid in making the diagnosis of vascular compromise. Despite discouraging appearances of the mucosa on endoscopy the surgeon should attempt to rescue the flap. Third, haematemesis should act as a warning sign for vascular compromise [13]. Fourth, if flap viability is in doubt urgent re-exploration is essential as it can save the flap. Finally, there is a need for close flap monitoring in the early postoperative period $[14,15]$. We have relied on a flap monitoring method combining clinical and instrumental tools as clinical methods alone are insufficient for early diagnosis of flap compromise. However, as such methods including a cutaneous window in the neck, or exteriorised segment of the jejunum have numerous drawbacks including positive predictive value of sixty-four, and false positive result of thirty-six percent [9], we suggest other post operative flap monitoring methods, such as a micro-dialysis probe, should be considered [16].

The patient reported here developed a late venous thrombosis in a free jejunal flap used to reconstruct a pharyngo-oesophageal defect. When recognised early these flaps can be rescued, with acceptable functional outcomes, by urgent re-exploration of the pedicle even at a late stage.

\section{REFERENCES}

[1] J. J. Disa and A. L. Pusic, "Reconstruction of the Hypo- pharynx with the Free Jejunum Transfer,” World Journal of Surgical Oncology, Vol. 94, No. 6, 2006, pp. 466-470. doi:10.1002/jso.20483

[2] B. Seidenberg, S. S. Rosenak, E. S. Hurwitt and M. L. Som, "Immediate Reconstruction of the Cervical Esophagus by a Revascularized Isolated Jejunal Segment," Annals of Surgery, Vol. 149, No. 2, 1959, pp. 162-171. doi:10.1097/00000658-195902000-00002

[3] D. M. McKee and C. R. Peters, "Reconstruction of the Hypopharynx and Cervical Esophagus with Microvascular Jejunal Transplant,” Clinics in Plastic Surgery, Vol. 5, No. 2, 1978, pp. 305-312. doi:10.1016/0002-9610(80)90197-X

[4] T. R. Hester, F. M. McConnel, F. Nahal, M. J. Jurkiewicz and R. G. Brown, "Reconstruction of Cervical Esophagus, Hypopharynx and Oral Cavity Using Free Jejunal Transfer,” American Journal of Surgery, Vol. 140, No. 4, 1980, pp. 487-491.

[5] D. E. Theile, D. W. Robinson and G. J. McCaffrey, "Pharyngolaryngectomy Reconstruction by Revascularized Free Jejunal Graft,” ANZ Journal of Surgery, Vol. 56, No. 11, 1986, pp. 849-852.

doi:10.1111/j.1445-2197.1986.tb01837.x

[6] C. K. Tsao and H. C. Chen, “Adequate Venous Drainage: The Most Critical Factor for a Successful Free Jejunal Transfer,” Annals of Plastic Surgery, Vol. 53, No. 3, 2004, pp. 229-234. doi:10.1097/01.sap.0000116286.61316.28

[7] J. J. Disa and P. G. Cordeiro, "Efficacy of Conventional Monitoring Techniques in Free Tissue Transfer: An 11Year Experience in 750 Consecutive Cases," Annals of Plastic Surgery, Vol. 104, No. 1, 1999, pp. 97-101. doi:10.1097/00006534-199907000-00013

[8] C. R. Bradford and R. M. Esclamado, "Monitoring of Revascularized Jejunal Autografts," Archives of Otolaryngology_Head \& Neck Surgery, Vol. 118, No. 10, 1992, pp. 1042-1044. doi:10.1001/archotol.1992.01880100032009

[9] B. C. Cho and D. P. Shin, "Monitoring Flap for Buried Free Tissue Transfer: Its Importance and Reliability,” Plastic and Reconstructive Surgery, Vol. 110, No. 5, 2002, pp. 1249-1258. doi:10.1097/00006534-200210000-00006

[10] J. A. Fearon and C. L. Cuadros, "Flap Failure after Microvascular Free-Tissue Transfer: The Fate of a Second Attempt," Plastic and Reconstructive Surgery, Vol. 86, No. 4, 1990, pp. 746-751. doi:10.1097/00006534-199010000-00024

[11] A. J. Ascioti and W. L. Hofstetter, "Long-Segment, Supercharged, Pedicled Jejunal Flap for Total Esophageal Reconstruction," Journal of Thoracic and Cardiovascular Surgery, Vol. 130, No. 5, 2005, pp. 1391-1398. doi:10.1016/j.jtcvs.2005.06.032

[12] H. C. Chen and B. K. Tan, "Behaviour of Free Jejunal Flaps after Early Disruption of Blood Supply," Annals of Thoracic Surgery, Vol. 73, No. 3, 2002, pp. 987-989. doi:10.1016/S0003-4975(01)03015-6

[13] A. Alaani, R. Hogg, S. S. Minhas, C. Jennings and A. P. Johnson, "Pseudoaneurysm after Total Pharyngolaryngectomy with Jejunal Graft Insertion: Two Different Presentations," European Archives of Otorhinolaryngology, Vol. 262, No. 4, 2005, pp. 255-258. 
doi:10.1007/s00405-004-0801-5

[14] G. Bertino and M. Benazzo, "Reconstruction of the Hypopharynx after Free Jejunum Flap Failure: Is a Second Free Jejunum Transfer Feasible?” Oral Oncology, Vol. 44, No. 1, 2008, pp. 61-64. doi:10.1016/j.oraloncology.2006.12.010

[15] H. Bafitis and J. O. Stallings, "A Reliable Method for Monitoring the Microvascular Patency of Free Jejunal Transfers in Reconstructing the Pharynx and Cervical Esopha- gus,” Plastic and Reconstructive Surgery, Vol. 83, No. 5, 1989, pp. 896-898.

doi:10.1097/00006534-198905000-00024

[16] M. Brix, P. Muret, B. Ricbourg and P. Humbert, "Monitoring Free Flaps with Cutaneous Microdialysis: Preliminary Study in Oral Cavity Reconstruction,” Revue de Stomatologie et de Chirurgie Maxillo-Faciale, Vol. 107, No. 6, 2006, pp. 460-464. doi:10.1016/S0035-1768(06)77088-7 